\title{
Effect of mare's milk prebiotic supplementation on the gut microbiome and the immune system following antibiotic therapy
}

\author{
MADIYAR NURGAZIYEV $V^{1,2,5, \vartheta}$, YERMEK AITENOV ${ }^{1,2,5}$, ZHANAGUL KHASSENBEKOVA $^{2}$, \\ SANIYA AKPANOVA ${ }^{3}$, KAIRAT RYSBEKOV ${ }^{3}$, SAMAT KOZHAKHMETOV ${ }^{1,2,4,5}$, \\ AYAULYM NURGOZHINA ${ }^{1,2,5}$, SHYNGGYS SERGAZY ${ }^{1,2,5}$, LAURA CHULENBAYEVA ${ }^{1,2,5}$, \\ ZHANNA OSPANOVA ${ }^{2}$, ALTYNAI TUYAKOVA ${ }^{1,2,5}$, NURISLAM MUKHAMBETGANOV ${ }^{4}$, \\ RASHIDA SATTYBAYEVA ${ }^{3}$, SALTANAT URAZOVA ${ }^{3}$, NAZYM GALYMGOZHINA ${ }^{3}$, \\ ANARA ZHUMADILOVA ${ }^{5}$, ALEXANDR GULYAEV ${ }^{1,2,5}$, ALMAGUL KUSHUGULOVA $^{1,2,5}$ \\ ${ }^{1}$ National Laboratory Astana, Nazarbayev University. Z05H0P9, Kabanbay Batyr Avenue 53, Block S1, Nur-Sultan, Kazakhstan. \\ Tel. +7-717-2-706521, ^email: madiyar.nurgaziyev@nu.edu.kz \\ ${ }^{2}$ Kazakhstan Society of Researchers of the Human Microbiome. Tawelsizdik 34, Z00T2C6, Nur-Sultan, Kazakhstan \\ ${ }^{3}$ Nur-sultan (Astana) Medical University. Beibitshilik Street 49/A, 010000, Nur-Sultan, Kazakhstan \\ ${ }^{4}$ SaumalBioTech LLP. Z05H0K9, Kabanbay Batyr Avenue 6/4, Nur-Sultan, Kazakhstan \\ ${ }^{5}$ Nazarbayev University. Kabanbay Batyr Avenue 53, 010000, Nur-Sultan, Kazakhstan
}

Manuscript received: 1 July 2020. Revision accepted: 8 October 2020

\begin{abstract}
Nurgaziyev M, Atenov Y, Khassenbekova Z, Akpanova S, Rysbekov K, Kozhakhmetov S, Nurgozhina A, Sergazy S, Chulenbayeva L, Ospanova Z, Tuyakova A, Mukhambetganov N, Sattybayeva R, Urazova S, Galymgozhina N, Zhumadilova A, Gulyaev A, Kushugulova A. 2020. Effect of mare's milk prebiotic supplementation on the gut microbiome and the immune system following antibiotic therapy. Biodiversitas 21: 5065-5071. Antibiotic treatment can severely affect the gut microbiome for short-term and longterm consequences. Probiotic and prebiotic supplements are widely prescribed to modulate the composition and function of the human gut microbiome. The current study aims to determine the impacts of mare's milk prebiotics on the diversity of gut bacterial communities and the local immune system when administered during and after a course of antibiotic therapy. Six children aged 4 to 5 years diagnosed with bilateral bronchopneumonia were prescribed cephalosporin (cefuroxime) antibiotics. During the 60 days of the study, three children consumed mare's milk prebiotics, while the other three did not. Fecal samples were collected daily during antibiotic therapy and every five days after the last day of antibiotic treatment. Total DNA was isolated, and the taxonomic composition of the gut microbiome was analyzed by sequencing the 16S rRNA gene (V1-V3 region). The MULTIPLEX MAP platform was used to evaluate the local immune status. The relative abundance of 11 genera was reduced and did not recover until the last day of the study. The abundance of Bacteroides was not significantly altered in either group. Christensenella, Rothia, Abiotrophia, Acinetobacter, Anaerotruncus, Holdemania, and Turicibacter numbers were significantly increased on day five and remained at the same level during the study period. Cephalosporin administration also reduced the levels of pro-inflammatory and anti-inflammatory cytokines/chemokines (MIP1 $\alpha$, TNF $\alpha$, GMCSF, GCSF, sCD40L, FGF2, TGF $\alpha$, IL1 $\alpha$, and IP10).
\end{abstract}

Keywords: Antibiotic treatment, immune system, mare's milk, microbiome

\section{INTRODUCTION}

Apart from their short-term effects on the human microbiome, antibiotics are known to have persistent effects for extended periods (Jernberg et al. 2010). Shifting of the human gut microbiome after antibiotic administration is known as 'dysbiosis' and can persist in patients for up to 2 years (Jernberg et al. 2007). Long-term alterations in the gut microbiome can lead to various diseases, such as obesity, allergies, antibiotic-associated diarrhea, inflammatory bowel disease (IBD), and numerous other health issues (Grazul et al. 2016). Antibiotic-induced changes in the microbiome can also have a profound impact on the immune system. Commensal microbial species that inhabit the intestinal tract are involved in the development, maintenance, and function of the immune system. Antibiotic administration causes alterations in the intestinal commensal bacterial population and modifies immune defenses, which in turn can have an impact on health (Ubeda and Pamer 2012).

Cephalosporins are broad-spectrum antibiotics frequently used in the clinic to treat various infections, such as skin, urinary tract, and respiratory tract infections (Tauzin et al. 2019). Cephalosporins are a class of $\beta$-lactam antibiotics derived from the fungus Acremonium and previously known as Cephalosporium. They are grouped into five generations based on their spectrum of coverage and different effects against pathogenic microorganisms causing respiratory diseases such as Streptococcus pneumoniae and hemophilic bacilli. Research on cephalosporins has increased interest in recent years. It is due to the increase in B-lactam pneumococcal resistance and the role of cephalosporins in the emergence of extended-spectrum B-lactamase (ESBL) Enterobacteriaceae (Tauzin et al. 2019). More importantly, cephalosporins create dysbiosis in the large intestine of mammals, which in 
turn allows the growth of antimicrobial-resistant Enterococcus faecium (Hendrickx et al. 2015). This study prescribed cefuroxime, which is a subgroup of secondgeneration cephalosporins to patients.

The definition of prebiotics has been revised many times. There is still an ongoing debate about what may be deemed a prebiotic. The concept of prebiotics may continue to evolve as our understanding of the microbiota role in host health continues to expand (Holscher 2017). The World Health Organization (WHO) defines prebiotics as nonviable food components that confer health benefits to the host associated with the modulation of the microbiota (Pandey et al. 2015). Prebiotics are often used to reduce the prevalence of diarrhea and relieve inflammation and other symptoms associated with IBD (Pandey et al. 2015). Several factors influence microbial colonization in the gut, and diet plays a vital role in modulating the composition and metabolism of the gut microbiome (Scott et al. 2013; Singh et al. 2017). The inclusion of prebiotics or probiotics in the diet may be a beneficial way to promote the development of beneficial microbiota and the inhibition of potential pathogens present in the human intestine (Markowiak and Ślizewska 2017). Mare's milk, which is also known as 'saumal' in Central Asia, is administered in these countries to prevent pulmonary tuberculosis, anemia, rickets, diabetes mellitus, obesity, and inflammatory disorders of the gastrointestinal tract (Kushugulova et al. 2018). Mare's milk differs widely from the milk of other dairy animals in terms of its components, such as milk fat and protein concentration. A previous study by Guri et al. (2015) showed that mare's milk has a lower casein concentration, but it contains twice whey than that of cow's milk. The antimicrobial activity of mare's milk is associated with whey fraction (Guri et al. 2015). The major components of mare's milk are oligosaccharides. Oligosaccharides prevent pathogenic adhesion to the intestinal mucosa (Uniacke-Lowe 2011). Lactobacilli and Bifidobacteria have prebiotic properties (Guri et al. 2015). They prefer oligosaccharides to be consumed and have immunomodulatory effects (Ding et al. 2017; Kusharyati et al. 2020). Mare's milk also contains biologically active compounds; lysozyme; lactoferrin; the vitamins A, B1, B2, B6, B9, B12, and C; and the trace elements $\mathrm{Na}, \mathrm{Ca}, \mathrm{K}, \mathrm{P}$, $\mathrm{Fe}, \mathrm{Mg}, \mathrm{Cu}, \mathrm{I}, \mathrm{S}, \mathrm{Si}, \mathrm{Zn}$, and C (Kozhakhmetov et al. 2020).

There is limited knowledge on how mare's milk influences the composition of the gut microbiota and the gut immune system during and after antibiotic treatment. This study, therefore, aimed to investigate the impacts of mare's milk on the diversity of gut bacterial communities in children who received cephalosporin antibiotics. The authors believe that the results of this study will help in the design of methodologies and therapeutic measures for the prevention of collateral antibiotic effects and provide some recommendations and suggestions for future studies.

\section{MATERIALS AND METHODS}

Ethical permission for this study was obtained from the local ethics committee at the National Laboratory of Astana (protocol No. 20 dated 09/22/2017, IORG0006963), and this study is registered on ClinicalTrials.gov under study identifier NCT03657836. Written informed consent was obtained from parents before any study-related procedure. Patients were admitted to City Children's Hospital No. 1, Nur-Sultan, Kazakhstan, in 2019.

\section{Study groups and sampling procedure}

The study consisted of six children aged 4 to 5 years diagnosed with bilateral bronchopneumonia. They are prescribed antibacterial cephalosporin class (cefuroxime) 40 grams per day from the $1^{\text {st }}$ to the $7^{\text {th }}$ day. It was diluted in warm water. The patients were divided into two groups. Group 1 (treatment group) was supplemented with mare's milk in parallel to the antibiotic treatment. Group 2 (control) was not supplemented with mare's milk prebiotics and received only antibiotic treatment. Group 1 received prebiotics daily until the end (day 60) of the study. The stool samples were collected daily from days 1 to 7 during the antibiotic treatment and every five days at the followup period to analyze the compositional and structural alterations in the gut microbiota. The sampling frequency was associated with the renewal cycle of intestinal epithelial cells. Stool samples were taken from patients according to the instructions provided by manufacturers. All samples were collected in sterile containers and stored at a temperature of $-80{ }^{\circ} \mathrm{C}$ prior to DNA extraction.

\section{DNA extraction, library preparation, and sequencing}

DNA was extracted using the QIAamp DNA Stool Mini Kit (Qiagen, Germany) and quantified with the dsDNA HS Qubit Assay Kit (Thermo Fisher, USA). It was measured using the Nanodrop 2000/2000 s Spectrophotometer and Qubit ${ }^{\circledR}$ 2.0 Fluorometer (Invitrogen, USA) and then stored at $-30^{\circ} \mathrm{C}$ before the library preparation process. Libraries were prepared using the Nextflex 16s V1-V3 Amplicon-seq Kit (Perkin Elmer, USA), suitable for Illumina platforms. Purified amplicons were pooled and sequenced on an Illumina MiSeq platform according to the manufacturer's standard instructions. In total, 85 stool samples were sequenced to an average sequence depth $<150000$ reads per sample. Cytokines and chemokines such as eotaxin, GCSF, GMCSF, sCD40L, IL1RA, IL8, MIP1 $\alpha$, MIP1 $\beta$, RANTES, TNF $\alpha$, TNF $\beta$, FGF2, TGF $\alpha$, PDGF-AA, IL3, IP10, VEGF, IL1 $\alpha$, and IL13 were evaluated using a human cytokine/chemokine magnetic bead panel included in a Milliplex MAP kit (Millipore Corp., MA, USA). Levels were measured with a Bio-Plex ${ }^{\circledR} 3 D$ suspension array system (Bio-Rad Laboratories, CA, USA).

\section{Bioinformatic analysis}

Bioinformatic analysis was performed in the $\mathrm{R}$ statistical language (v.3.4.4). The Divisive Amplicon Denoising Algorithm 2 (DADA2, v.1.8.0) was used for quality filtering, cleaning, and trimming the raw sequencing data. Reads with an abundance of $<0.001 \%$ of the total number of reads were removed. The Phyloseq package (v.1.24.2) was used to construct taxonomic distributions at the phylum and genus levels. Analysis of alpha diversity was carried out to assess the abundance of a 
community (Chao 1 and Ace indices) and calculation of biodiversity (Shannon index) (McMurdie and Holmes 2013). All graphs were generated using ggplot2 (v.3.0.0). The Maaslin2 tool was used to analyze multivariate associations between microbial abundances and immune response biomarkers.

\section{Statistical analysis}

The Shapiro-Wilk normality test and exact WilcoxonMann-Whitney test were used to compare Shannon index comparison groups at individual time points. Linear regression models of variation in alpha diversity across sampling times were compared using one-way ANOVA. The influence of individual parameters, such as patient sex and age, on relative biodiversity, was determined using ANOSIM and PERMANOVA statistical tests. The BETADISPER test was used to test for random dispersion and validate beta-diversity statistics. Pearson correlation coefficients were calculated for associations between immune response biomarkers and microbial abundances. Q-values were adjusted for multiple comparisons using the Benjamini-Hochberg false discovery rate correction with a threshold of $\mathrm{q}<0.25$.

\section{RESULTS AND DISCUSSION}

\section{Contrasting changes in taxon richness and diversity}

All patients had a positive clinical response to antibiotic treatment at the time of discharge from the clinic. It was indicated by leveling of intoxication symptoms, improvements in appetite and sleep, and normalization of body temperature, as well as normalization of markers of inflammation in the blood $(\mathrm{ESR}=8.2 \mathrm{~mm} / \mathrm{hour}$ vs. 17.6 $\mathrm{mm} /$ hour; CRP level=1.7 $\mathrm{mg} / \mathrm{L}$ vs. $19.0 \mathrm{mg} / \mathrm{L}$; leukocytes $=8.5 * 10^{9}$ cells $/ \mathrm{L}$ vs. $16.4 * 10^{9}$ cells $\left./ \mathrm{L}\right)$.

We examined 112 stool samples in total collected from the MM (Mare's milk-administered patients) $(\mathrm{N}=51)$ and $\mathrm{AB}$ (patients who received standard antibiotic treatment) $(\mathrm{N}=61)$ groups. Samples were collected daily during antibiotic therapy from day 1 to day 7 and then every five days and last for 60 days. As expected, cephalosporin treatment triggered profound gut bacterial depletion and shifts in microbiome composition.

The analysis of taxon richness (Chao1, ACE) and diversity (Shannon) was applied to both groups (control and mare's milk treatments). Statistical tests (Figure 1) showed that there was an impact on $\alpha$ diversity. As shown in the graphs, the $\alpha$-diversity regression line slope increased from day 1 until day 60 in the mare's milk group, whereas it decreased in the control group (ANOVA, $\mathrm{p}<0.05$ ). (A) Time-series patterns of alpha-diversity metrics in the control and MM (Mare's milk-administered patients) treatment groups. Straight lines represent linear regression. Alpha indices increased over time in the $\mathrm{MM}$ treatment group but not in the control group (ANOVA, $\mathrm{p}<0.05^{*}$ ). (B and C) Beta diversity of the microbiota of samples from MM treatment group and control group. Ordination of the microbiome data using principal component analysis (PCoA) on weighted (B) and unweighted (C) UniFrac distances. (D and E) Beta diversity of the microbiota of $A B$ treatment samples during and after a course of antibiotics. Ordination of the microbiome data using principal component analysis (PCoA) on weighted (D) and unweighted (E) UniFrac distances.

Analysis using sample identity, sampling type, and time point as additional covariates on the level of beta diversity (restricted to postantibiotic (day 6+) samples) showed significant $(\mathrm{P}<0.001)$ separation between $\mathrm{MM}$ samples. Significant differences were observed for unweighted UniFrac analysis (Figure 1B and D) based on the ANOSIM $(\mathrm{R}=0.2328 ; \quad \mathrm{p}=0.001) \quad$ and PERMANOVA (F.model=5.3916; R2=0.0908; $\mathrm{p}=0.001$ ) tests. The results of weighted UniFrac (Figure 1C and E) were also significantly different by the ANOSIM ( $\mathrm{R}=0.3319$; $\mathrm{p}=0.001$ ) and PERMANOVA tests (F.model=10.622; $\mathrm{R} 2=0.1644 ; \mathrm{p}=0.001$ ).

Noticeable changes in the microbiome were detected on the 2nd day of antibiotic therapy, and composition fluctuations continued after completing antibiotic treatment until the 60th day of the study. Due to the small number of observations, the revealed changes in taxonomic characteristics are mostly insignificant.
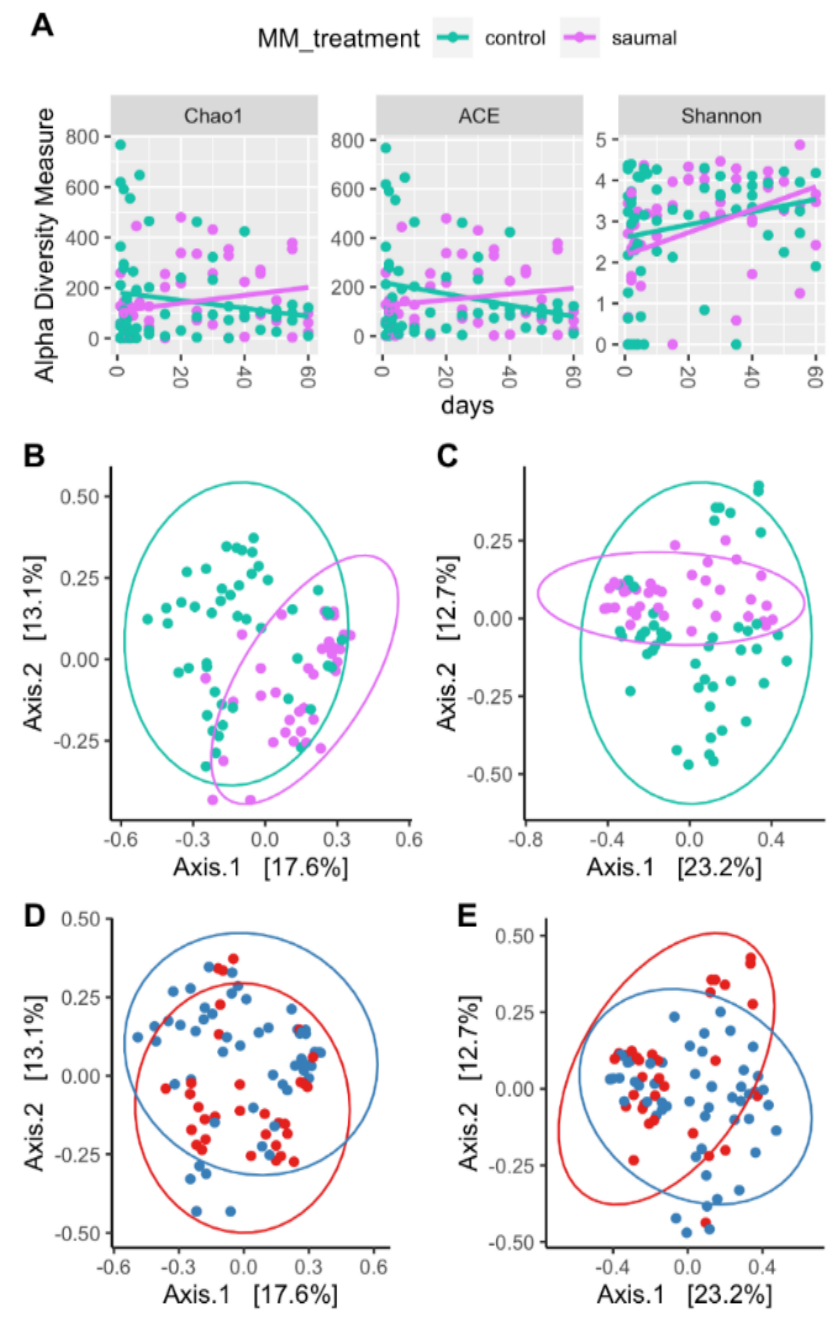

AB_treatment $\rightarrow$ AB $\rightarrow$ none

Figure 1. Alpha-diversity metrics and beta-diversity analysis of $\mathrm{MM}$ and $\mathrm{AB}$ samples 


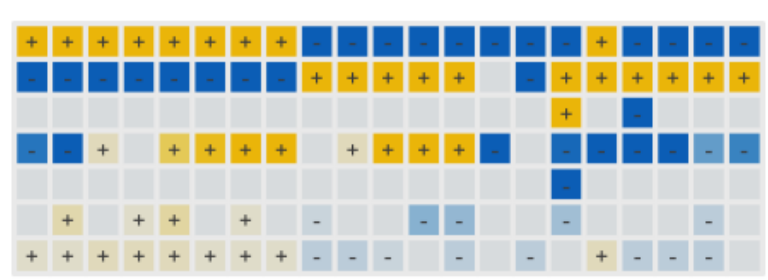

Firmicutes; Lachnoclostridium Unclassified OTU_1188 Unclassified OTU_570

Firmicutes; Streptococcus Firmicutes; Abiotrophia Bacteroidetes; Bacteroides Firmicutes; Lachnospiraceae

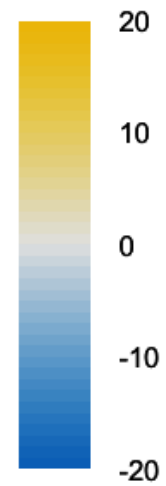

Figure 2. Microbial taxa associated with up/downregulation of immune response biomarkers. The scale bar represents the negative log2 of the correlation significance value (q.val) multiplied by the coefficient of correlation. Only significant associations are presented

At the genus level, we observed that the genera Ezakiella, Finegoldia, Fusobacterium, Facklamia, Gordonibacter, Jonquetella, Mobiluncus, Negativicoccus, Peptoniphilus, Peptostreptococcus, and Porphyromonas were reduced entirely in the $\mathrm{AB}$ group on the $2^{\text {nd }}$ day of cephalosporin treatment and did not fully recover until the 60th day of the study. Representatives of the genera Acidaminococcus, Anaerococcus, Anaerostipes, Blautia, Butyricimonas, Coprobacter, Gemmiger, Odoribacter, Parabacteroides, Paraprevotella, Parasutterella, Pseudoflavonifractor, and Veillonella decreased in abundance three days after starting antibiotic therapy but recovered by the 10th day of the study. The genera Sutterella, Akkermansia, Alistipes, Bacteroides, Eggerthella, Flavonifractor, Fusicatenibacter, Intestinimonas, Oscillibacter, Phascolarctobacterium, Ruminococcus, and Barnesiella mildly fluctuated. Their numbers were significantly reduced on the first days of antibiotic therapy. However, they recovered quickly after discontinuation of cephalosporin treatment. Treatment of broad-spectrum antibiotics drastically reduced the populations of Dialister and Megasphaera, which did not fully recover following cessation of antibiotic therapy until the last day of the study.

In contrast, the numbers of the genera Christensenella (Firmicutes, Clostridia), Rothia (Actinobacteria, Actinobacteria), Abiotrophia (Firmicutes, Bacilli), Acinetobacter (Proteobacteria, Gammaproteobacteria), Anaerotruncus (Firmicutes, Clostridia), Holdemania (Firmicutes, Erysipelotrichia), and Turicibacter (Firmicutes, Erysipelotrichia) were significantly increased by the fifth day of antibiotic therapy. It remained at the same level throughout the study period.

\section{Correlations between the abundance of specific taxa and the cytokine/chemokine immune response profile}

Antibiotic therapy led to significant decreases in the levels of anti-inflammatory cytokines/chemokines. TGFa in healthy subjects strengthens the proliferation of enterocytes and induces intestinal adaptation. We found in this study that TGF $\alpha$ levels were decreased significantly (pvalue $=0.006846)$ after antibiotic therapy. The pro- inflammatory cytokines/chemokines were reduced considerably on the 3rd day of antibiotic therapy (Table 1). The pro-inflammatory cytokines/chemokines were MIP1 $\alpha$ $(\mathrm{p}=0.00455), \quad \mathrm{TNF} \alpha \quad(\mathrm{p}=0.004719), \quad$ and $\mathrm{GCSF}$ (Granulocyte-Colony Stimulating Factor). GCSF is also known as colony-stimulating factor 3. A downward trend for GCSF was observed from day 3 to day 60 (3rd day: $\mathrm{p}=0.02052$; 20th day: $\mathrm{p}=0.05179$ ). The level of GMCSF (Granulocyte-Macrophage Colony-Stimulating Factor, also known as a colony-stimulating factor) also decreased significantly from day 20 until the end of the study (20th day: $p=0.05426$; 60th day: $p=0.03894$ ). A decrease in the $\mathrm{sCD} 40 \mathrm{~L}$ level $(\mathrm{p}=0.02494)$ was also observed.

There were also reductions in anti-inflammatory cytokine/chemokine levels after antibiotic therapy. On day 3, FGF2 (p=0.04573) and IP10 $(p=0.04256)$ were significantly reduce. TGF $\alpha(p=0.0002572)$ and IL1 $\alpha$ $(p=0.01124)$ were significantly changed on day 20 , and decreasing trends persisted on day 60 for TGF $\alpha$ $(p=0.0005257), \operatorname{IL} 1 \alpha(p=0.0195)$, and VEGF $(p=0.05866)$.

The correlation of the relative abundance of specific microbial OTUs (Operational taxonomic units) in the samples with an increase or decrease in cytokine/chemokine levels was analyzed using multivariate association analysis (Figure 2). The results revealed several OTUs, i.e., four OTUs were classified as the Firmicutes phylum, one OTU was identified as the Bacteroidetes taxa, and the other two OTUs were unclassified. Further classification to a lower taxon level showed that the OTU genera are Lachnospiraceae, Abiotrophia, Streptococcus, Lachnoclostridium, and Bacteroides.

Heat maps show that significant increases or decreases in the level of specific cytokines/chemokines were associated with changes in the relative abundance of several OTUs. IL-8 level was strongly (more saturated color) negatively correlated with the abundances of Lachnoclostridium and Streptococcus but less correlated (i.e., less saturated color) with Lachnospiraceae and strongly positively correlated with that of OTU_1188. The same principle applies to the other biomarkers shown in Figure 2. 
Table 1. Cyto/Chemokine levels in $\mathrm{AB}$ and $\mathrm{MM}$ samples at different stages of the study

\begin{tabular}{|c|c|c|c|c|c|c|c|c|c|}
\hline \multirow{2}{*}{$\begin{array}{l}\text { Cytokine/ } \\
\text { chemokine } \\
\text { (pg/mL) }\end{array}$} & \multirow{2}{*}{$\begin{array}{l}\text { Before } \\
\text { antibiotic } \\
\text { therapy }\end{array}$} & \multicolumn{4}{|c|}{$\mathbf{A B}$} & \multicolumn{4}{|c|}{ MM } \\
\hline & & 3 day & 10day & 20 day & 60 day & 3 day & 10day & 20 day & 60 day \\
\hline Eotaxin & $2.9 \pm 0.0$ & $2.9 \pm 0$ & $3.5 \pm 0.3$ & $3 \pm 0.2$ & $2.9 \pm 0$ & $3.2 \pm 0.2$ & $2.9 \pm 0.1$ & $4.1 \pm 1.3$ & $2.9 \pm 0.1$ \\
\hline GCSF & $1.3 \pm 0.2$ & $0.6 \pm 0.1 *$ & $5.5 \pm 3.9$ & $0.8 \pm 0.1 *$ & $0.8 \pm 0.4$ & $3.5 \pm 2.4$ & $0.7 \pm 0$ & $85.9 \pm 80.6$ & $5.2 \pm 4.4$ \\
\hline GMCSF & $4.4 \pm 1.3$ & $2.5 \pm 1.4$ & $3.8 \pm 0.4$ & $1.7 \pm 0.1 *$ & $0.8 \pm 0.8 *$ & $8.1 \pm 0.6$ & $1.6 \pm 0.7$ & $11.1 \pm 7.7$ & $6.1 \pm 2.9$ \\
\hline sCD40L & $2.6 \pm 0.2$ & $2.4 \pm 0.2$ & $3.2 \pm 1.2$ & $2.2 \pm 0.3$ & $1.7 \pm 0.2^{*}$ & $3.1 \pm 0$ & $2.1 \pm 0.2$ & $30.3 \pm 27.7$ & $178.1 \pm 176$ \\
\hline IL1RA & $28.8 \pm 14.6$ & $1 \pm 0.1$ & $2 \pm 0.7$ & $0.8 \pm 0.3$ & $1.1 \pm 0.2$ & $1.8 \pm 0.5$ & $1.7 \pm 0.4$ & $27.4 \pm 26.5$ & $76.7 \pm 75.1$ \\
\hline IL8 & $2.7 \pm 0.0$ & $2.7 \pm 0$ & $1.9 \pm 0.5$ & $1.8 \pm 0.8$ & $3.3 \pm 0.6$ & $2.8 \pm 0$ & $2.6 \pm 0$ & $3 \pm 0.3$ & $3.5 \pm 0.8$ \\
\hline MIP1 $\alpha$ & $3.7 \pm 0.2$ & $3.1 \pm 0 *$ & $4.5 \pm 0.3^{*}$ & $3.6 \pm 0.4$ & $3 \pm 0$ & $3.1 \pm 0$ & $3.1 \pm 0$ & $3.3 \pm 0.2$ & $9.3 \pm 6.2$ \\
\hline MIP1 $\beta$ & $23.8 \pm 3.6$ & $23.5 \pm 2.9$ & $36.9 \pm 10.3$ & $18.3 \pm 6.3$ & $19.1 \pm 4.9$ & $41.8 \pm 14.1$ & $13.9 \pm 6.5$ & $37.3 \pm 10.4$ & $141.2 \pm 130.4$ \\
\hline RANTES & $0.2 \pm 0.0$ & $0.2 \pm 0$ & $0.2 \pm 0$ & $0.2 \pm 0$ & $0.2 \pm 0$ & $0.2 \pm 0$ & $0.2 \pm 0$ & $0.2 \pm 0.1$ & $0.2 \pm 0$ \\
\hline $\mathrm{TNF} \alpha$ & $3.3 \pm 0.3$ & $2.4 \pm 0.1 *$ & $5.2 \pm 0.8$ & $2.7 \pm 0.3$ & $1.8 \pm 0.4$ & $2.8 \pm 0.4$ & $2.4 \pm 0.2$ & $5.5 \pm 3.2$ & $31.2 \pm 28.9$ \\
\hline $\mathrm{TNF} \beta$ & $1.4 \pm 0.0$ & $1.5 \pm 0$ & $1.9 \pm 0.2$ & $1.5 \pm 0.2$ & $1.5 \pm 0$ & $1.5 \pm 0$ & $1.9 \pm 0.2$ & $1.5 \pm 0.2$ & $1.5 \pm 0$ \\
\hline FGF2 & $3.6 \pm 1.1$ & $1.1 \pm 0.2 *$ & $2.3 \pm 0.6$ & $1.3 \pm 0.5$ & $1.5 \pm 0.7$ & $1.6 \pm 0$ & $1.4 \pm 0.3$ & $96.1 \pm 94,8$ & $5.5 \pm 4.3$ \\
\hline TGF $\alpha$ & $2.83 \pm 0.0$ & $2.8 \pm 0$ & $2.9 \pm 0.1$ & $2.8 \pm 0 *$ & $2.8 \pm 0 *$ & $3 \pm 0.1$ & $2.8 \pm 0$ & $3.2 \pm 0.4$ & $2.8 \pm 0.1$ \\
\hline PDGFAA & $1.9 \pm 0.0$ & $1.9 \pm 0$ & $3.4 \pm 0.6$ & $2 \pm 0.2$ & $1.9 \pm 0$ & $2 \pm 0$ & $1.9 \pm 0$ & $2.1 \pm 0.2$ & $2 \pm 0.1$ \\
\hline IL3 & $3.2 \pm 0.5$ & $2.7 \pm 0.1$ & $3.8 \pm 0.9$ & $4.6 \pm 2.4$ & $6.8 \pm 4.1$ & $5.6 \pm 1.3$ & $1.9 \pm 0$ & $6.5 \pm 4.3$ & $22.3 \pm 20$ \\
\hline IP10 & $27.5 \pm 1.9$ & $22.2 \pm 1.5$ & $42.8 \pm 8.2$ & $34 \pm 17.1$ & $19.2 \pm 10.6$ & $39.7 \pm 5.7$ & $22.4 \pm 2.1$ & $47.3 \pm 16$ & $24.3 \pm 6$ \\
\hline VEGF & $3.4 \pm 1.4$ & $1 \pm 0.1$ & $24.5 \pm 23.1$ & $2.2 \pm 1.7$ & $0.7 \pm 0.4$ & $5.3 \pm 4$ & $0.5 \pm 0.1$ & $0.8 \pm 0.1$ & $0.6 \pm 0.2 *$ \\
\hline $\mathrm{IL} 1 \alpha$ & $413 \pm 53.7$ & $319.3 \pm 37.4$ & $518 \pm 186.6$ & $159.8 \pm 42.8 *$ & $195.9 \pm 42.1^{*}$ & $461.9 \pm 109$ & $686.1 \pm 30$ & $812.3 \pm 330.8$ & $721.7 \pm 280.2$ \\
\hline IL13 & $2.4 \pm 0.0$ & $2.3 \pm 0.1$ & $4.6 \pm 1.2$ & $2.7 \pm 0.5$ & $2.3 \pm 0$ & $3.4 \pm 0.5$ & $2.2 \pm 0.1$ & $6.8 \pm 4.5$ & $2.3 \pm 0.2$ \\
\hline
\end{tabular}

\section{Discussion}

Based on the results, we observed some significant alterations in particular members of the microbial community. Even though there were no significant changes in taxonomic characteristics identified, we observed remarkable decreases in 11 genera at the compositional level. Therefore, these genera did not fully recover until the last day of the study, among others the reductions of Fusobacterium and Facklamia abundance. Previous studies showed that Fusobacterium species could be related to various diseases, such as Lemierre's syndrome, sepsis, and respiratory tract infections (Afra et al. 2013; Han Yiping 2015). Facklamia species are Gram-positive cocci. They are rarely reported as a cause of clinical infection. Facklamia can be associated with invasive diseases such as urinary tract infections, chorioamnionitis, and infective endocarditis (Malone et al. 2017; Rahmati et al. 2017). The results also showed that Bacteroides abundance was not significantly altered by cephalosporin. It supports previous findings investigating the effect of cephalosporins on the intestinal microbiota (Burdet et al. 2019; Rashid et al. 2015). Apart from a decrease in the abundance of several genera, there was an increase in Christensenella, Rothia, Abiotrophia, Acinetobacter, Anaerotruncus, Holdemania, and Turicibacter. The role of these microorganisms is poorly understood; however, some of them are recognized as opportunistic pathogens. For example, it has been reported that the genus Rothia is implicated in severe respiratory diseases, such as pneumonia and acute bronchitis, and members of this genus mostly affect immunocompromised hosts (Maraki and Papadakis 2015).

This study showed that the MM and AB groups were significantly different. Based on the UniFrac distances, we observed a significant overlap in the microbiota composition between day three and day 7 of the study.
Samples collected on days 5, 10, 20, and 60 days tended to be grouped based on UniFrac distances (Wilcoxon criterion, $\mathrm{p}<0.001$ for comparing distances between subjects and distances between other matters). After completing the course of antibiotic therapy, the biodiversity index on day 10 was 3.0. This index remained in the range of 2.8-3.6 until the end of the study. The changes caused by mare milk supplementation in patients can be observed by comparing the two treatment groups. The overall results indicated a tendency toward increasing diversity in the gut microbiota of patients receiving mare's milk supplementation.

The multiplex platform was used in this study because it covers a broad spectrum of chemokines and cytokines involved in the immune response. Based on the results, there was a high correlation between cytokine synthesis and OTU variants representing Lachnoclostridium, Streptococcus, and a yet unclassified OTU_1188 strain. The coefficients of these correlations possessed a "plus" sign in some cases and a "minus" sign in others. The significant OTUs representing bacteria of the genus Lachnoclostridium share a common active role in dietderived fiber degradation, leading to the production of short-chain fatty acids (SCFAs) by anaerobic fermentation. The presence of commensal bacteria OTU 83 and OTU 1168, Lachnoclostridium, and OTU_1188 in the microbiota had a high correlation with cytokine synthesis. They could be an additional diagnostic criterion when analyzing the state of local intestinal immunity. The presence of Lachnoclostridium in the gut microbiota was associated with the production of PDGF-AA, FGF, IL3, VEGF, MIP1 $\alpha$, MIP1 $\beta$, RANTES, eotaxin, and IgA.

The OTU-1188 strain had a negative correlation with the group mentioned above of cytokines and, in turn, supplemented this series with the synthesis of the following 
cytokines: GMCSF, IL1 $\alpha$, TGF $\alpha$, IL1r $\alpha$, IL13, IP10, IgG1, IL8, TNF $\beta$, MIP1 $\alpha$, and $\mathrm{sCD} 40 \mathrm{~L}$, all of which were negatively correlated with Lachnoclostridium. MIP1 $\alpha$ was the only biomarker for which these two strains had a common positive correlation. MIP1 $\alpha$ was a chemokine in the CC family (beta chemokine receptors). The Streptococcus parasanguinis CC87K strain had its scale of positive and negative correlations with the biomarkers shown in the heat map. Regarding the Bacteroides and Lachnospiraceae genera, the diagram shows a weaker correlation than that of the Lachnoclostridium strain. Such a relationship is characteristic of both positive and negative correlations. Thus, the intestinal commensal bacteria correlate with immunoregulatory biomarkers of the immune system and is involved in maintaining intestinal homeostasis (Shi et al. 2017).

Cytokine production between the two groups was significantly different by day 60 (Table 1). Upon mare's milk administration, there was an increase in the production of the cytokines IL 3, MIP1 $\beta$, IL1Ra, Il1a, and SCD40L compared to the production at the beginning of antibiotic treatment. The synthesis of these cytokines was closely correlated with constituents of the intestinal commensal bacteria, Lachnoclostridium, and Streptococcus bacteria. sCD40L was additionally associated with the unclassified OTU_570 strain. Positive effects of mare's milk administration affected the production of proinflammatory cytokines IL $1 \alpha$ and IL1r $\alpha$. IL1r $\alpha$ is known to be a specific IL1 $\alpha$ receptor antagonist. In the body, the balance between these two cytokines plays a vital role in protecting the body from infection while limiting further tissue damage. IL1ro is an endogenous anti-inflammatory agent, and an increase in its level correlates with a favorable prognosis in the human immune system (Ageeva et al. 2011).

At the beginning of antibiotic therapy, the ratio between the IL1 $\alpha$ and IL1r $\alpha$ cytokines was at the level of $1: 14.3$. However, the ratio increased to $1: 178$ in the $A B$ group and decreased to 1:9.4 in the MM group on day 60 . The results demonstrate the positive effect of mare's milk on the immune system of children who received antibiotic therapy. The balance between IL1r $\alpha$ and IL1 $\alpha$ in the AB group did not recover after antibiotic therapy, even on day 60 . The balance is significantly skewed in favor of the proinflammatory cytokine. Local changes in the cytokine balance in the intestinal immune system in favor of increasing pro-inflammatory cytokine levels indicate the preservation of inflammatory processes in the intestine and dysbiotic disorders in the microbiota. It is known that an imbalance in cytokine production leads to various pathological conditions, allergy, autoimmune diseases, and infections (Simbirtsev 2019). The study on the local immune balance in the intestine showed that the administration of mare's milk contributed to the restoration of the cytokine balance after an inflammatory process in the body and antibiotic therapy. Data collected from patients during the inflammatory process and intoxication symptom presentation showed a decrease in the balance between the cytokines IL1r $\alpha$ and IL1 $\alpha$ relative to the initial ratio support an argument that mare's milk possesses immunoprotective properties. Therefore, it can contribute to improving the immunoresistance of the body.

Antibiotic-induced disruption of the gut microbiota remains a global issue, and probiotic and prebiotic supplementation is one way to prevent the adverse health effects of antibiotics. This study demonstrated alterations in the gut microbiome at the taxonomic level after cephalosporin therapy. There were significant reductions in the abundances of 11 genera, which did not recover until the last day of the study. Cephalosporin therapy reduced the levels of pro-inflammatory and anti-inflammatory cytokines/chemokines (MIP1 $\alpha$, TNF $\alpha$, GMCSF, GCSF, sCD40L, FGF2, TGF $\alpha$, IL1 $\alpha$, and IP10). The study also showed that mare's milk supplementation is useful for improving the diversity of gut bacterial communities and has the effects on the immune system and cytokine/chemokine response during and after a course of cephalosporin treatment. Although this study had an obvious limitation due to the relatively small number of participants, the results demonstrated evident changes in the gut microbiota composition and immune system within seven days of antibiotic therapy and during the follow-up period.

\section{ACKNOWLEDGMENTS}

This study was supported by scientific grants (AP05135073, AP05134659, and AP09058099) from the Ministry of Education and Science of Kazakhstan.

\section{REFERENCES}

Afra K, Laupland K, Leal J, Lloyd T, Gregson D. 2013. Incidence, risk factors, and outcomes of Fusobacterium species bacteremia. BMC Infect Dis 13 (1): 1. DOI: 10.1186/1471-2334-13-264.

Ageeva E, Shtygasheva O, Riazantseva N. 2011. Population association features of interleukine gene polymorphism in khakases with gastroduodenal diseases. Ter Arkh 83 (2): 16-19.

Burdet C, Grall N, Linard M, Bridier-Nahmias A, Benhayoun M, Bourabha K, Magnan M, Clermont $\mathrm{O}$, d'Humières $\mathrm{C}$, Tenaillon $\mathrm{O}$, Denamur E, Massias L, Tubiana S, Alavoine L, Andremont A, Mentré F, Duval X. 2019. Ceftriaxone and cefotaxime have similar effects on the intestinal microbiota in human volunteers treated by standard-dose regimens. Antimicrob Agents Chemother 63 (6): 1-12. DOI: 10.1128/AAC.02244-18.

Ding YH, Qian LY, Pang J, Lin JY, Xu Q, Wang LH, Huang DS, Zou H. 2017. The regulation of immune cells by Lactobacilli: A potential therapeutic target for anti-atherosclerosis therapy. Oncotarget 8 (35): 59915-59928. DOI: 10.18632/oncotarget.18346.

Grazul H, Kanda LL, Gondek D. 2016. Impact of probiotic supplements on microbiome diversity following antibiotic treatment of mice. Gut Microb 7 (2): 101-114. DOI: 10.1080/19490976.2016.1138197.

Guri A, Paligot M, Crèvecoeur S, Piedboeuf B, Claes J, Daube G, Corredig M, Griffiths MW, Delcenserie V. 2015. In vitro screening of mare's milk antimicrobial effect and antiproliferative activity. FEMS Microbiol Lett 363 (2): 1-7. DOI: 10.1093/femsle/fnv234.

Han Yiping. 2015. Fusobacterium nucleatum: a commensal turned pathogen. Curr Opin Microbiol 1 (23): 141-147. DOI: 10.1080/10810730902873927.Testing.

Hendrickx APA, Top J, Bayjanov JR, Kemperman H, Rogers MRC, Paganelli FL, Bonten MJM, Willems RJL. 2015. Antibiotic-driven dysbiosis mediates intraluminal agglutination and alternative segregation of Enterococcus faecium from the intestinal epithelium. MBio 6 (6): 1-11. DOI: 10.1128/mBio.01346-15. 
Holscher HD. 2017. Dietary fiber and prebiotics and the gastrointestinal microbiota. Gut Microb 8 (2): 172-184. DOI 10.1080/19490976.2017.1290756.

Jernberg C, Löfmark S, Edlund C, Jansson JK. 2007. Long-term ecological impacts of antibiotic administration on the human intestinal microbiota. ISME J 1 (1): 56-66. DOI: 10.1038/ismej.2007.3.

Jernberg C, Löfmark S, Edlund C, Jansson JK. 2010. Long-term impact of antibiotic exposure on the human intestinal microbiota. Microbiology 156 (11): 3216-3223. DOI: 10.1099/mic.0.040618-0.

Kozhakhmetov S, Babenko D, Nurgaziyev M, Tuyakova A, Nurgozhina A, Muhanbetganov N, Chulenbayeva L, Sergazy S, Gulyayev A, Saliev T, Kushugulova A. 2020. The combination of mare's milk and grape polyphenol extract for treatment of dysbiosis induced by dextran sulfate sodium. Biodiversitas 21 (5): 2275-2280. DOI 10.13057/biodiv/d210558.

Kusharyati DF, Pramono H, Ryandini D, Manshur TABU. 2020. Bifidobacterium from infant stool: the diversity and potential screening. Biodiversitas 21 (6): 2506-2513. DOI: 10.13057/biodiv/d210623.

Kushugulova A, Kozhakhmetov S, Sattybayeva R, Nurgozhina A, Ziya A, Yadav H, Marotta F. 2018. Mare's milk as a prospective functional product. Funct Foods Health Dis 8 (11): 548. DOI: 10.31989/ffhd.v8i11.528.

Malone L, Cm MBA, Grigorenko E, Stalons D. 2017. Id Week 2015. 2 (September), 2633851. DOI: 10.1093/o.

Maraki S, Papadakis IS. 2015. Rothia mucilaginosa pneumonia: A literature review. Infect Dis 47 (3): 125-129. DOI: 10.3109/00365548.2014.980843.

Markowiak P, Ślizewska K. 2017. Effects of probiotics, prebiotics, and synbiotics on human health. Nutrients 9 (9). DOI: 10.3390/nu9091021.

McMurdie PJ, Holmes S. 2013. Phyloseq: An R Package for Reproducible Interactive Analysis and Graphics of Microbiome Census Data. PLoS ONE 8 (4): e0061217. DOI: 10.1371/journal.pone.0061217.
Pandey KR, Naik SR, Vakil BV. 2015. Probiotics, prebiotics and synbiotics- a review. J Food Sci Technol 52 (12): 7577-7587. DOI: 10.1007/s13197-015-1921-1.

Rahmati E, Martin V, Wong D, Sattler F, Petterson J, Ward P, Butler-Wu SM, She RC. 2017. Facklamia species as an under-recognized pathogen. Open Forum Infect Dis. DOI: 10.1093/ofid/ofw272

Rashid MU, Rosenborg S, Panagiotidis G, Söderberg-Löfdal K, Weintraub A, Nord CE. 2015. Ecological effect of ceftarolineavibactam on the normal human intestinal microbiota. Antimicrob Agents Chemother 59 (8): 4504-4509. DOI: 10.1128/AAC.00530-15.

Scott KP, Gratz SW, Sheridan PO, Flint HJ, Duncan SH. 2013. The influence of diet on the gut microbiota. Pharmacol Res 69 (1): 52-60. DOI: $10.1016 /$ j.phrs.2012.10.020.

Shi N, Li N, Duan X, Niu H. 2017. Interaction between the gut microbiome and mucosal immune system. Military Med Res 4 (1): 17. DOI: $10.1186 / \mathrm{s} 40779-017-0122-9$.

Simbirtsev A.S. 2019. Immunopharmacological aspects of the cytokine system. Bulletin of Siberian Medicine. 18 (1): 84-95 DOI: 10.20538/1682-0363-2019-1-84-95.

Singh RK, Chang HW, Yan D, Lee KM, Ucmak D, Wong K, Abrouk M, Farahnik B, Nakamura M, Zhu TH, Bhutani T, Liao W. 2017. Influence of diet on the gut microbiome and implications for human health. J Translational Med 15 (1): 1-17. DOI: 10.1186/s12967-0171175-y.

Tauzin M, Ouldali N, Béchet S, Caeymaex L, Cohen R. 2019. Pharmacokinetic and pharmacodynamic considerations of cephalosporin use in children. Expert Opin Drug Metab Toxicol 15 (11): 869-880. DOI: 10.1080/17425255.2019.1678585

Ubeda C, Pamer EG. 2012. Antibiotics, microbiota, and immune defense. Trends Immunol 33 (9): 459-466. DOI: 10.1016/j.it.2012.05.003.

Uniacke-Lowe T. 2011. Studies on Equine Milk and Comparative Studies on Equine and Bovine Milk Systems. [Dissertation]. University College Cork, UK. 
